\title{
Chemical biology: fats as research subjects
}

\author{
Vivien Marx
Fats add structure, they signal, they interact. In the lab, lipids are tough to work with but worth the challenge.

\begin{abstract}
"You're fat" can be hurtful to hear. "They're just fats" is hurtful, too, especially to the ears of biochemists. It implies that fats don't matter much. Prejudices about lipids have been common, says Anne-Claude Gavin, a biochemist at the European Molecular Biology Laboratory (EMBL) in Heidelberg. Lipids have long been considered as the mere brick and mortar that separate interior from exterior in plant and animal cells and as less interesting because they don't encode information in the enticing way that nucleic acids do.

One of many physiological findings about lipid-protein interaction is that lipid modifications regulate some proteins generated by cancer genes, says Rami Hannoush, a chemical biologist at the biopharmaceutical company Genentech. Around five percent of coding genes are involved in lipid metabolism and lipid homeostasis, and around one-third of cellular proteins are associated with the cell membrane, says Gavin. In eukaryotic cells, lipids are building materials as well as signaling molecules. They are, she says, abundant, diverse cellular metabolites with variable chemical scaffolds, head-groups, fatty acid lengths, branching and degrees of saturation.

"Lipids are very challenging to deal with in terms of their behavior," says Hannoush. Lipids stick to surfaces, and nonspecific lipid binding can deliver false-positive

Genomic information sheds light on the diversity of proteins, including isoforms and splice variants; but no such reference exists for lipids, says Gavin. Yet many researchers see more reasons to embrace fats than to shun them. They aim to advance methods development for lipids research, and want to grow the community and dispel prejudices. They study lipid signaling pathways and how lipids modify and regulate proteins, analyze lipid populations in samples or track spatial distribution of lipids.
\end{abstract} assay results. Specialized techniques are needed to handle and solubilize lipids, he says. They cannot be studied directly by using genetic methods, says biochemist Carsten Schultz, formerly at EMBL and now at Oregon Health and Science University. Many experiments require organic chemistry, "a notoriously slow process," he says.

\section{Making numbers talk}

To explore lipids in action, the Gavin lab developed the high-throughput liposome microarray-based assay (LiMA) ${ }^{1}$. It lets scientists quantify and image many types of protein-lipid interactions. It measures lipids recruited to liposomes, balls of artificial membranes that are cell membrane

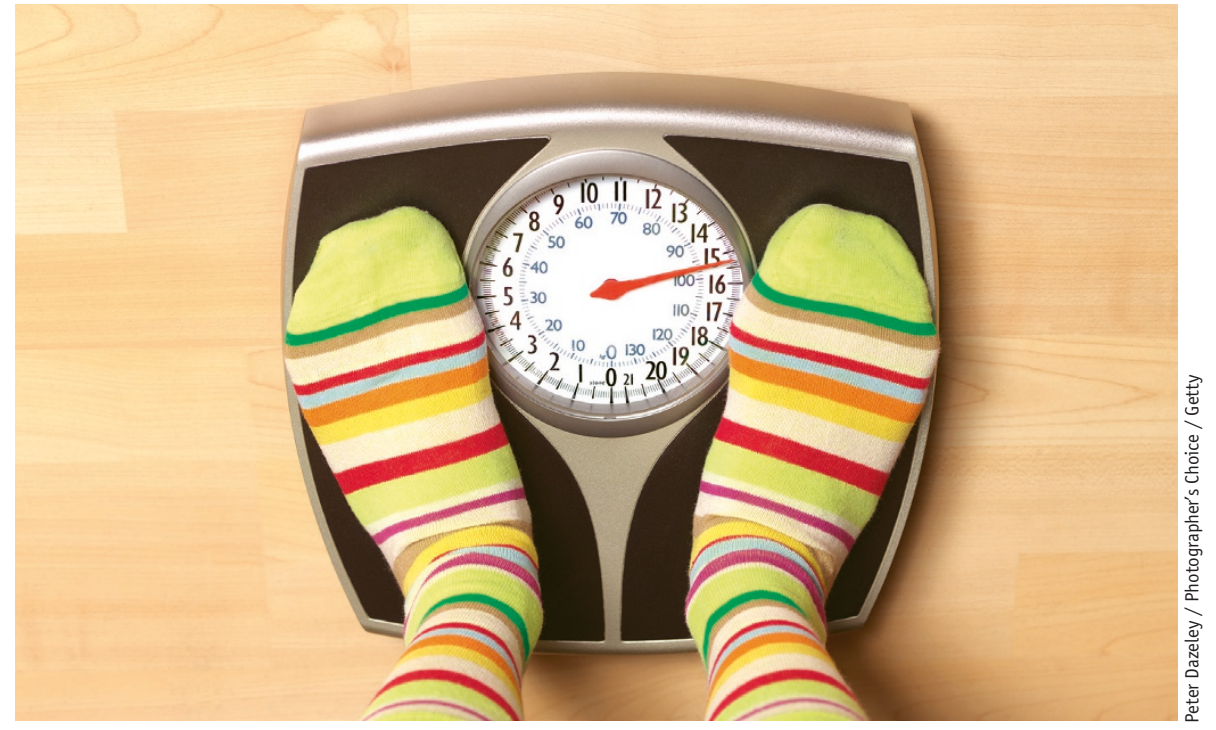

Fats can be worrisome. In the lab, fats are welcome subjects of study.

surrogates. The team is developing "more mature" membranes, to mimic, for example, the membranes of endoplasmic reticulum, Golgi or mitochondria. The researchers also want to use LiMA to characterize lipid function, to connect protein-binding affinity to downstream enzymatic activity. LiMA detects subtleties: varying protein affinities to different chemical compositions of membrane. Perhaps stronger membrane-binding increases activity. "Sometimes it's not that they bind or don't bind, but they bind slightly more or slightly less," says Gavin.

The team does science plus engineering: they make the devices, including microfluidics, and they build the interfaces between microfluidics, biochemistry assays, imaging and data analysis. Gavin hopes to commercialize LiMA. Her group arrays the lipids onto the chips, but a company's production pipeline would avoid 
batch variation. When labs contact Gavin, they often send samples for LiMA-based analysis. When results are promising, she offers the researchers a stint in her lab to optimize how they use LiMA. Lipids research needs tools, and this is a way to popularize the technology, she says.

\section{Happy birthday, probe}

Some lipids attach to proteins for minutes, others hang on for hours; this varies by protein, and the resulting protein behavior can vary, too, says Hannoush. Among the many lipid-based modifications to proteins are palmitoylation and myristoylation, which involve attachment of different types of fatty acids ${ }^{2}$. There is also prenylation, in which different-sized hydrophobic groups tag a protein. Given their roles in signaling cascades, fatty acylation and prenylation intrigue labs working in translational research. But, he says, one method for studying lipid-based modifications of proteins sorely needed updating to avoid the hazards and cost of working with radioactivity. To replace the use of radiolabeled fatty acids that are metabolically integrated into the cell, he and colleagues developed nonradioactive probes for detecting fattyacylated proteins in vitro and in vivo. The method is celebrating a ten-year anniversary.

These probes are fatty acid or isoprenoid analogs with reactive groups that can be labeled with a fluorophore or conjugated to biotin. As Hannoush explains, the readout signal is quick: assays take less than a minute instead of three weeks, and the probes enable at least a 30,000-fold improvement in detection sensitivity when compared to radiolabeled analogs. Using these probes, labs can explore whether a protein is lipid modified and study lipidation states in disease.

The method's first generation involved a few hours of incubation to harness the cell's own biosynthetic machinery for incorporating the probe, and the next generation of probes made it possible to study the lipidation status of proteins of interest, says Hannoush. Next up is work to use click chemistries and tagging approaches for live-cell imaging and detection. "This is a high-bar challenge," he says.

Hannoush has also developed a fluorescence imaging method to track palmitoylated Wnt proteins in single cells. He and his team use a bio-orthogonal chemical probe combined with in situ proximity ligation.

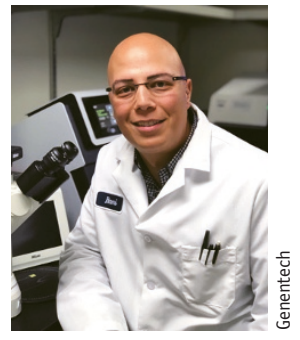

Lipid modifications regulate some proteins generated by cancer genes, says Genentech's Rami Hannoush. tissue. It lipidates proteins such as Wnt and does so with the help of a kink in its hydrocarbon chain. Hannoush and colleagues determined through structural biology techniques that the protein's receptors have U-shaped cavities into which palmitoleic acid fits precisely because of the kink. This recognition mechanism evolved for mediating specificity between Wnt and its receptors.

OSHU's Schultz is keen on detecting lipid-based modifications in intact, live cells and understanding the dynamics of these modifications. He and colleagues recently developed live-cell probes for visualizing lipids, specifically the signaling molecules sphingosine and diacylglycerol ${ }^{3}$. These 'trifunctional lipids' are caged lipid derivatives that act as photochemical probes for exploring many aspects of a single lipid species in live cells and for following lipid signaling across a cell population. The trifunctionality of these probes relates to their two photoreactive groups for caging and photo-crosslinking and an alkyne for enabling click chemistry.

Given their low abundance in cells, sphingosine and diacylglycerol are tough lipids to track. Raising lipid levels by overexpressing metabolic enzymes is slow and risks confounding because other lipid levels are raised along with the one of interest. By equipping probes with caging groups, lipid levels can be raised in a targeted way, 'in a flash'. The probe is inactive and resists the cell's processing until activated by light. The lab applies a second photochemical reaction to cross-link the probe to the proteins the lipid interacts with. The complex can then be characterized by mass spectrometry or other methods.

The researchers used the probe to localize lipids in cell lines modeling the often-fatal Niemann-Pick disease, in which lipids accumulate in cells instead of being trafficked and metabolized. They tested the probe on the cells of three patients with mild to severe cases of this disorder to start exploring when and where in the cell lipid trafficking hits a bottleneck.

Schultz continues methods development for studying lipids in live cells, organoids and tissues. In his view, research with model systems or with isolated, often artificial, membranes will help to unravel principles important to the lipid research community. Then these data need to be correlated with information about other biomolecules, such as proteins, nucleotides and sugars. That is when "pure in vitro models sometimes do not suffice," he says.

\section{Lipid mass spec}

Labs use mass spectrometry to identify and quantify lipids and lipid-protein interactions on a systems-wide level. Oliver Fiehn of the University of California at Davis and colleagues point out that liquid chromatography-mass spectrometry (LC-MS) is widely used for measuring concentrations of lipid species, as well as other metabolites, in samples. But many factors influence both number and nature of detected lipids. Fiehn says he and his lab were "a bit shocked" when they reviewed nearly 200 lipidomics papers and found that almost half of them described direct infusion of the sample instead of an initial chromatographic separation of analytes ${ }^{4}$. Over the past few years, he has been glad to see a drop in the use of direct infusion.

Fiehn urges the community to do quantification with many internal lipid standards. LC-MS techniques have increased in speed and efficiency, but issues crop up when comparing work between labs using different instruments. Contributing factors could be varying operating conditions or the lack of standards for LC-MS-based untargeted lipidomics. It would be helpful to use

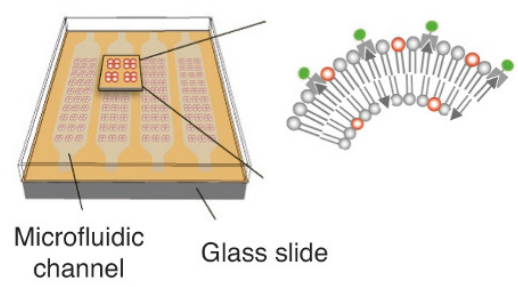

The high-throughput liposome microarray-based assay (LiMA) is for exploring lipids in action. 


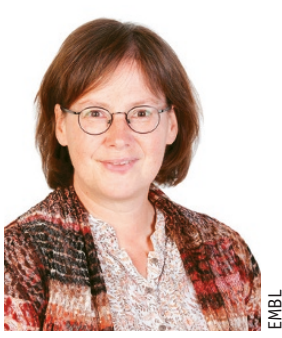

Around five percent of coding genes are involved in lipid metabolism and lipid homeostasis, says Anne-Claude Gavin.

reference standards for each target lipid species, but many lipids are either unavailable or too costly. Experiments also involve many variables, such as varying peak intensities, even within a lipid class, which depend on solvent mixtures when the electrospray is ionized. Results can also differ between reverse-phase liquid chromatography and normal-phase liquid chromatography.

In a more recent ring trial, Fiehn and colleagues compared lipidomic analysis on multiple MS instruments including models by Agilent, Thermo, Leco and Sciex. They used identical blood samples from people who had just eaten a meal ${ }^{5}$. In each case, the team kept sample preparation methods, chromatography and data processing the same and compared output across different platforms. Labs choose the instruments they want, says Fiehn, but internal standards, ultraperformance liquid chromatography conditions or the columns used can at least in principle be standardized. In this experiment, the researchers note that all platforms revealed the "top-hit discriminating lipid species" in the samples and the estimated concentrations of lipids obtained were all highly comparable. Fiehn and his team were happy to see different mass spectrometers deliver such comparable results: he now believes that lipidomics is mature enough for use in 'precision medicine.'

When performing the analyses, the researchers say it's important to avoid saturating both the instrument's ion source and the detector. To do so, it's helpful to evaluate linear dynamic ranges using dilutions and also to adjust the resuspension volume or injection volume before running the samples. They highlight this aspect because, says Fiehn, some scientists hope to copy and paste a method from one machine to another. "You can't, unless these methods are really on identical machines," he says. "Instead, small adaptations are always needed, not difficult, but peak saturation should be very much avoided."

Fiehn and his group participated in another inter-lab lipidomics comparison that included 31 labs around the world. "Ring trials are the best way to go forward in validating technologies," he says. In this trial, each lab used its own method, which made for a diverse set of lipidomics workflows. Each lab measured 1,527 lipids in samples of Standard Reference Material 1950-Metabolites in Frozen Human Plasma (SRM 1950). They also compared results to those of a previous trial with SRM 1950 measured by the LIPID MAPS Consortium.

Among other results, the sum of common lipids identified in the LIPID MAPS study was $30 \%$ higher than this trial's sum. The authors note "significant discrepancies for specific classes between both studies," whose causes have yet to be explored. The study authors also point to the lack of a clear community-wide consensus about quantifying lipids and note that this is a polarizing subject within the community, with "methodological and philosophical differences to consider."

\section{Chasing isomers}

One challenge in lipid quantification and analysis is that the mammalian cell lipidome contains many isomeric lipid species. Chemist Yu Xia, formerly at Purdue University and now at Tsinghua University, developed a way of quantifying differences in isomer compositions of several fatty acid and phospholipids species between healthy and cancer tissue ${ }^{6}$. Lipid isomers have the same mass but appear to fulfill different functions, such that her method of finding and counting unsaturated lipid isomers can provide a functional snapshot of a cellular lipidome. As Xia explains, the isomers can differ in the location of the carbon-carbon $(\mathrm{C}=\mathrm{C})$ double bonds. With tandem MS (MS/MS) methods, especially low-energy collision-induced dissociation (CID), it's hard to generate fragment ions because the $\mathrm{C}=\mathrm{C}$ locations have high bond-dissociation energies. Some methods address this,

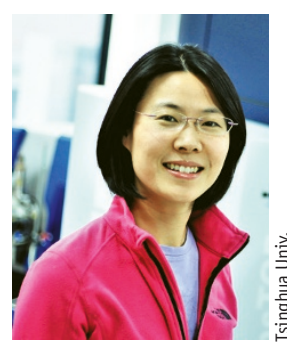

but their caveats include the need for large sample amounts or specialized instruments.

Xia developed a different way to locate this $\mathrm{C}=\mathrm{C}$ bond by coupling a photochemical reaction-the Isomeric lipid species are worth finding and quantifying, says Yu Xia.

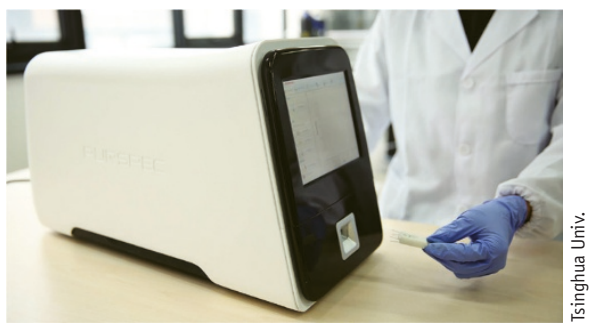

The mini mass spec could be used for fast lipid profiling.

In doing so she and her team could identify fatty acid C18:1 and other complex lipids that contain fatty acyl 18:1. Their measurements with rodent tissue showed elevated levels of some isomers in tumor tissue, a hint of the technique's possible biomedical applications, she says.

Xia had spent a few years exploring a mass-spec-based way to distinguish biomolecular isomers. The $\mathrm{PB}$ reaction is a cycloaddition reaction that specifically modifies the $\mathrm{C}=\mathrm{C}$ bonds in lipids. When the products of the $\mathrm{PB}$ reaction are ionized, they are susceptible to being cleaved at the $\mathrm{C}=\mathrm{C}$ locations, she says. With $\mathrm{PB}-$ MS/MS, labs can use lower-energy CID mass spec instruments to analyze lipids and find isomers. It's not hard to apply this method: labs just need a UV lamp, a fused silica capillary for the electrospray ionization (ESI) line, and acetone as the ESI co-solvent.

Xia is happy to see others picking up her approach because as more groups find $\mathrm{C}=\mathrm{C}$ bonds, more data for comparing results will be available. She also sees a need for data analysis software to simplify the way early-adopter labs and the larger lipidomics community share results. In collaboration with the Blanksby lab at the Queensland University of Technology in Brisbane, Xia is setting out to compare results obtained using $\mathrm{PB}$ and a different ion-dissociation method, ozone-induced dissociation (OzlD), developed in the Blanksby lab.

Xia also collaborates with Zheng Ouyang, another Tsinghua researcher (and her husband), to implement PB and OzID on a miniaturized mass spec instrument that Ouyang has been developing. This could potentially bring mass spec into the clinical setting for diagnosis and disease monitoring, says $\mathrm{Xia}^{7}$. She and Ouyang are developing methods to handle small sample sizes, such as one microliter of plasma or micrograms of tissue, for lipid analysis. 


\section{Mini mass spec}

Just like the large instruments, the mini mass spec measures mass-to-charge ratio and can handle similar mass ranges, says Ouyang. The goal is to use mass spec without sample prep or chromatography-based purification. Instead, people will use disposable kits with a cartridge and do direct analysis of lipids in blood or tissues. It's intended for general users without mass spec training.

The system applies ambient ionization, and samples are ionized in their original, untreated states, says Ouyang. For mass analysis, the lipid ions are transferred into an ion-trap mass analyzer in a vacuum system. It was challenging to find the most efficient way to extract lipids from a sample. As he re-engineered the mass spec instrument to miniaturize it, he had to assure it had adequate abundance of ions in the vacuum to achieve good sensitivity without the traditional, elaborate pumping system. He developed and tested several ambient ionization methods to enable cartridge-based analysis.

Because lipid concentrations in biological tissues are relatively high, lipid analysis is an attractive application for the mini mass spec, says Ouyang. The instrument can help with finding and measuring lipid isomers. The isomer ratios could be used as biomarkers with accurate quantification without needing internal standards. He and his team have incorporated the $\mathrm{PB}$ reaction into tissue analysis with the mini mass spec and tested it with tissue from diabetes patients.

Ouyang began working on the mini mass spec project a decade ago. His Purdue University graduate school mentor, analytical chemist and mass spectrometrist

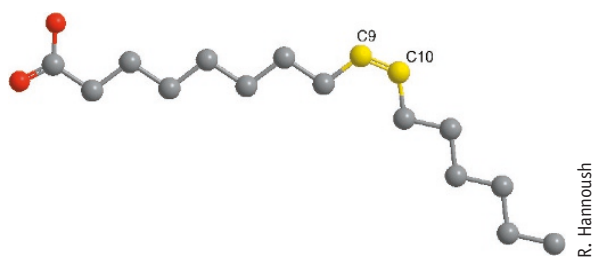

Palmitoleic acid lipidates proteins such as Wnt with the help of a kink (yellow) in its hydrocarbon chain.

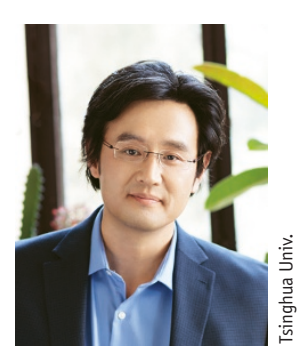

Trying mass analysis with a mini mass spec sounded insane to many, says Zheng Ouyang.
Graham Cooks, imprinted the idea on him. The process involved overcoming technical and mental challenges, says Ouyang. Attempting mass analysis traditionally done with huge instruments in one the size of a shoebox "sounded insane" to many, he says. The instrument has moved beyond the prototype stage and commercialization is underway. Purspec Technologies, the start-up he cofounded, will collaborate with Purdue and Tsinghua Universities to continue instrument development.

Interactions with biologists asking him for advice have added motivation, says Ouyang. In his view, the reasons that mass spec instruments have not made inroads beyond analytical labs are their price and size and the fact that well-trained chemists are needed to run them. The mini mass spec will not replace standard instruments, which are key to original discovery in lipids research, but it could be used for fast screening in which the instrument's direct sampling ionization method could deliver a lipid profile from raw tissue.

\section{Seeing lipid worlds}

Chemical imaging approaches are another way to detect and measure biomolecules in tissue, and one of them is imaging mass spec, also called mass spec imaging, originally developed by Vanderbilt University researcher Richard Caprioli. The method has been applied to lipids, so some labs hope it can help with studying lipid dynamics. "The beauty with imaging mass spec is that you can really localize your lipids," says Gavin. It's slow and doesn't deliver subcellular resolution, but for analyzing lipids in a complex cellular context such as liver or muscle tissue, it reveals how lipid composition may differ within tissues related to health or disease. "That would be very difficult to do by other biochemical methods," she says, including classic mass spec, because one would need to average results from millions of cells.

Imaging mass spec will be part of the single-cell analysis repertoire, says Schultz, telling researchers which lipids are present in a single cell under particular conditions. "The closer we get to subcellular resolution, the more exciting the data will become," he says. Integrating biochemical with optical methods promises to help labs describe cell and tissue dynamics, he says. Down the line, labs will want to perform integrated lipid analysis with samples from both animal models and people.

When researchers detect a lipid in a particular environment, they tend to see it as one signal, says Schultz, but it is more likely a signal from a combination of lipids. Labs will also want to obtain more detailed information, such as how far a lipid headgroup sticks out of the cell membrane, which is tough to measure since it's a matter of a fraction of a nanometer. "Further, lipid dynamics in cell membranes need to be determined," he says. He began his journey into lipids research by looking at phosphoinositides, which are phospholipids involved in many plasma membrane signaling pathways and which affect a range of cellular processes. These days, he says, any and all lipids have his interest: "I think we need to understand the lipid world, not just a single or group of species."

Vivien Marx is technology editor for Nature Methods (v.marx@us.nature.com)

1. Saliba, A.E. et al. Nat. Methods 11, 47-50 (2014).

2. Hannoush, R. et al. Nat. Chem. Biol. 6, 498-506 (2010).

3. Höglinger, D. et al. Proc. Natl. Acad. Sci. USA 114, 1566-1571 (2017).

4. Cajka, T. Trends Anal. Chem. 61, 192-206 (2014).

5. Bowden, J. et al. J. Lipid Res. 58, 2275-2288 (2017).

6. Ma, X. et al. Proc. Natl. Acad. Sci. USA 113, 2573-2578 (2016).

7. He, M. et al. Anal. Chem. 87, 2236-2241 (2015). 